\title{
A PADRONIZAÇÃO DO PROCESSO COMO PRIMEIRO PASSO PARA A IMPLEMENTAÇÃO DO DESENVOLVIMENTO LEAN DE PRODUTOS - EVIDÊNCIAS DA INDÚSTRIA TÊXTIL
}

\author{
Ana Julia Dal Forno ${ }^{1}$, Fernando Antonio Forcellini ${ }^{2}$, Fernando Augusto Pereira ${ }^{3}$, Liane Mählmann Kipper ${ }^{3 ;}$ Maurício Johnny Loos $^{2}$ \\ ${ }^{1}$ Professora das Engenharias, Universidade Federal de Santa Catarina (UFSC), Campus Blumenau, Blumenau/SC - Brasil \\ ${ }^{2}$ Programa de Pós Graduação em Engenharia de Produção, Universidade Federal de Santa Catarina (UFSC), Florianópolis/SC - \\ Brasil \\ ${ }^{3}$ Programa de Pós Graduação em Sistemas e Processos Industriais, Universidade de Santa Cruz do Sul (UNISC), Santa Cruz do Sul - \\ Brasil
}

*E-mail: anajudalforno@hotmail.com

\section{RESUMO}

A abordagem enxuta enfatiza que a padronização é a base para começar, ou seja, é preciso a estabilidade dos processos e das pessoas. No entanto, na prática percebe-se que muitas empresas querem ser "lean" esquecendo-se dessa preparação inicial. Assim, esse artigo tem o objetivo de analisar a padronização de processo em termos de bibliometria, desperdícios associados, indicadores e as práticas enxutas do desenvolvimento associadas a ela. A metodologia utilizada foi uma revisão sistemática da literatura, seguido de survey com as sete empresas têxteis que desenvolvem produtos no Brasil. Os resultados apontam que no desenvolvimento de produtos a padronização do processo ainda é uma prática que precisa ser mais explorada no meio empresarial e acadêmico, pois nem sempre aparece de forma direta e a falta de entendimento do seu conceito, dos benefícios e problemas podem gerar desperdícios que impactam no escopo do projeto e consequentemente na usabilidade.

Palavras-chave: Têxtil. Padronização. Desenvolvimento de produtos. Abordagem enxuta

\section{Introdução}

O padrão é o ponto de partida da melhoria. Isso significa que é necessário definir com clareza e precisão a maneira como se executam as ações. Isso ajuda a reduzir as variações que desestabilizam o sistema, comprometem os resultados e criam o "caos". Mas o que se deve ter em mente é que o padrão é simplesmente a melhor maneira de se fazer algo no momento presente e não para sempre. Se houver maneira mais adequada, esta deve passar a ser o padrão Ferro [7].

Para Dennis [6], o alicerce da produção é o padrão - aquilo que deve acontecer. $\mathrm{O}$ alicerce da excelência é adotar um padrão. Contudo, para muitos, o termo "padrão" sugere enormes manuais de baboseiras ilegíveis. Na abordagem enxuta, um padrão é uma imagem clara de uma condição desejada; são importantes porque tornam as anormalidades imediatamente visíveis para que ações corretivas possam ser tomadas. Um bom padrão é simples, claro e visual. Já Maçaneiro, Catczu e Korchaque [9], concluem que a padronização do conhecimento é importante na organização de processos administrativos. Neste estudo os autores desenvolveram uma ferramenta que veio sanar pontos falhos no departamento de vendas na área comercial da empresa estudada, por meio da padronização. No sistema lean os padrões estão ligados à ação.

$\mathrm{Na}$ manufatura o conceito de Trabalho Padronizado já está bem difundido e aplicado com sucesso. No entanto, ainda há lacunas ao adaptar para o desenvolvimento de produtos. Existe uma linha de pensamento que defende que interfere na criatividade dos funcionários, que torna o trabalho repetitivo e monótono. Porém, segundo Dennis [6], os benefícios são:

- Estabilidade de processos - diretamente relacionado com a consistente disponibilidade dos 4 Ms (Material, Máquina, Método, Mão-de-obra), ou seja, materiais e máquinas precisam estar disponíveis quando forem necessárias; o método de trabalho precisa ser consistente e uniforme e a mão-de-obra disponível e treinada adequadamente.

- Conhecimento do processo - identificação dos tempos das atividades e pontos de paradas, o que possibilita saber se está adiantado ou atrasado e se há algum problema;

- Aprendizagem organizacional - as experiências são compartilhadas, se um funcionário sai o conhecimento não fica perdido;

- Envolvimento das pessoas - fornece a base para as 
melhorias kaizen, integrando a equipe. As pessoas ficam mais motivadas.

- Treinamento - fornece uma base para o treinamento fornecendo-lhes planilhas, gráficos e aplicando na prática e no dia-a-dia o formato do trabalho padronizado.

Nesse contexto, esse artigo tem o objetivo de identificar se é feita a padronização de processos pelas empresas têxteis como primeiro passo para introduzir a abordagem enxuta no desenvolvimento de produtos. Assim, após a introdução, a seção 2 aborda definições sobre a importância da padronização e os tipos existentes. O capítulo 3 apresenta a bibliometria sobre padronização identificando em que ano as publicações começaram a aparecer, em quais países ela é mais citada e quais as práticas que são aplicadas em conjunto. Após, na seção 4 é apresentada a metodologia survey com as sete empresas têxteis, destacando a padronização, os indicadores utilizados e os desperdícios identificados no Processo de Desenvolvimento de Produtos (PDP). As conclusões finalizam as discussões com críticas quanto à utilização da padronização e seu uso, indicando pesquisas futuras.

\section{Revisão de Literatura}

Essa seção destaca a padronização sobre dois aspectos - sua relevância perante o PDP e os tipos de padronização, sendo de processo, projeto e habilidades. $O$ foco é na padronização de processos. A teoria também aborda a relação da padronização com a variabilidade.

\subsection{A importância da padronização}

Já afirmavam Rozenfeld et. al. [13], que a dificuldade de descrever como é o PDP tem reflexos importantes na forma como ele pode ser gerenciado. Devido à sua complexidade o primeiro passo para o gerenciamento eficiente do PDP é tornálo "visível" a todos os atores envolvidos. Os autores chamaram a padronização de modelagem de processos, na qual obter um modelo de PDP significa descrever as atividades, recursos, informações, fases, responsabilidades e outras possíveis dimensões do processo.

Ao documentar e disseminar o PDP de uma empresa, a gerência está definindo um padrão de como desenvolver seus produtos. Esse padrão pode ser aplicado pelas equipes e pelo gerente de cada projeto. Eles adaptarão as práticas descritas no modelo do processo conforme as necessidades do projeto. O PDP sistematizado e documentado permite que as particularidades de cada projeto e equipe de desenvolvimento sejam atendidas e, ao mesmo tempo, garante-se a utilização das melhores práticas de projeto e um linguajar padronizado e único para toda a coorporação. Isso aumenta a qualidade e repetibilidade dos projetos de desenvolvimento de produtos e, para que o processo-padrão possa ser reutilizado por várias pessoas, ele é documentado na forma de um modelo Rozenfeld et. al. [13].
Conforme Rozenfeld et. al. [13], de uma maneira geral, as empresas não possuem uma visão unificada do processo. Além disso, o desempenho de cada unidade da empresa e a diferença de escopo dos produtos não permitem que se tenha uma visão única do processo de desenvolvimento de produtos. Muitas vezes, cada pessoa possui um entendimento próprio do processo e utiliza um vocabulário e formalismo particular para descrevê-lo, advindos de sua área específica. No dia-a-dia, essas limitações acarretam problemas e ineficiências no PDP, dificultando a comunicação e integração entre os profissionais e as áreas envolvidas.

Segundo Perin [12], o processo quando padronizado pode alcançar altos níveis de qualidade e de produtividade uma vez que o resultado final é a obtenção de reprodução sistemática de uma "melhor prática" para a atividade. A padronização assegura que cada operador no processo produtivo saiba o que fazer, como fazer e quando fazer, Chaves [2].

A gestão por processos engloba o melhor entendimento das necessidades dos funcionários e clientes, facilidade de conduzir melhorias e clareza do controle econômico, sendo que a padronização dos procedimentos de trabalho reduziu os custos em 25\%, Palmberg [11].

A padronização da informação é uma das principais estratégias usadas no Sistema Produto-Serviço (PSS - Product Service System) para a reutilização do conhecimento e ser bem sucedidos nas abordagens de codificação, Morgan e Liker [10].

\subsection{Tipos de Padronização}

Para Morgan e Liker [10], a padronização é a base para reduzir as variabilidades através de listas de verificações e como um mecanismo para capturar o conhecimento. A padronização do projeto envolve o produto, seus componentes, matéria-prima e sua arquitetura. A padronização dos processos envolve tarefas comuns, sequência e duração das tarefas e a padronização das habilidades técnicas está relacionada com a capacidade das pessoas envolvidas na equipe do desenvolvimento.

O grande desafio do desenvolvimento lean de produtos é reduzir as variações preservando a criatividade. A variabilidade é um fator primário determinante do desempenho pobre do desenvolvimento de produtos. Existem dois tipos variabilidade de tarefas e variabilidade de chegada de demanda inter-relacionada. $\mathrm{O}$ primeiro tipo refere-se às diferenças nos métodos e na duração de tarefas específicas presentes na maior parte do desenvolvimento de produtos. Já a variação de chegada de demanda inter-relacionada refere-se a diferença de tempo entre o prazo marcado para a chegada da tarefa na estação de trabalho e sua chegada efetiva. Essa diferença é geralmente causada pelo primeiro tipo de variação, bem como por suas restrições de capacidade, Morgan e Liker [10].

Os mesmos autores sugerem que para minimizar a variabilidade de tarefa é preciso: 
- Adotar a padronização

rigorosa (processo/projeto/habilidades);

- Maximizar componentes compartilhados por plataformas (reuso);

- Matrizes de qualidade de componente;

- Arquitetura de plataforma modular;

- Conjunto alinhado de metas e objetivos específicos.

Para minimizar a variação de chegada de demanda interrelacionada, [10] recomendam:

- Minimizar a variação de tarefa;

- Rigorosa disciplina de programação;

- Padronização detalhada do PDP;

- Minimizar hand-offs (passagem de responsabilidades);

- Minimizar as mudanças de engenharia;

- Reduzir os tempos de ciclo de administração.

Focando na padronização de processo, um processo padronizado de desenvolvimento significa:

- Padronizar tarefas comuns;

- Padronizar sequências de tarefas;

- Padronizar a duração de tarefas.

Um PDP padronizado contribui significativamente para a comunicação mais precisa e maior entendimento as áreas funcionais.

\section{Bibliometria sobre Padronização}

A revisão sistemática da literatura foi realizada nas bases de dados Isi Web of Knowledge e Emerald Insight no dia 21/11/2013. As palavras-chaves utilizadas foram: Lean, New Product Development, Lean Product Development, Benchmarking, Lean Design, Benchmarking, Performance Measures e suas combinações. Na primeira base os filtros utilizados foram por tipo de documento (artigo), idioma (inglês), áreas de pesquisa e categorias (Management, Engineering Industrial, Operations Research Management Science, Engineering Manufacturing, Business, Engineering Mechanical, Engineering Multidisciplinary). Na Emerald Insigh a busca avançada considerou somente periódicos. Em ambas foi feita a seleção por título e resumo. A amostra de artigos considerada válida foi de 330 .

As principais perguntas a serem respondidas nessa etapa foram:

- Em que ano que a padronização do PDP teve seu auge?

- Quais as práticas do PDP enxuto que são aplicadas em conjunto com a padronização?

- Em quais países a prática da padronização está mais evidente?
Dos 330 artigos, 134 deles abordam a prática de padronização (41\%). A Figura 1 mostra que antes de 2007 havia poucas publicações que tratavam sobre essa prática no PDP. A partir de então ocorreu o auge até 2010. Isso está alinhado com o mesmo período que as empresas começaram a aplicar a abordagem enxuta nesse processo, tanto que desde 2012 essa prática já não foi mais mencionada.

Convém lembrar que a padronização identificada nos artigos analisados não foi classificada quanto a projeto, habilidade ou processo e que o foco desse artigo é para a padronização de processo.

Em relação à localização das aplicações (país ou continente) foram identificados que há três que se destacam - Estados Unidos, Reino Unido e Suécia, que juntos representam $40 \%$ dos trabalhos. A Tabela 1 detalha a contagem, sendo que houve trabalhos que aplicaram em mais de um local.

Quando verificado quais as práticas associadas à padronização, ela raramente aparece de forma isolada. Somente em dois trabalhos isso ocorreu, o que significa 1\%; seguido de duas práticas (padronização e outra) há 13 trabalhos (10\%). A Tabela 1 mostra a prática mais associada com a padronização é a rede de aprendizado.

Tabela 1. Práticas do PDP enxuto identificadas na literatura

\begin{tabular}{l|l|l}
\hline \multicolumn{2}{c}{ Prática Quantidade } & \multicolumn{2}{l}{} \\
\hline Rede Aprendizado & 82 & $16 \%$ \\
\hline Integração / Engenharia Simultânea & 76 & $15 \%$ \\
\hline Voz do Consumidor & 72 & $14 \%$ \\
\hline Envolvimento Inicial do Fornecedor & 66 & $13 \%$ \\
\hline Modularidade & 42 & $8 \%$ \\
\hline Engenheiro Chefe & 42 & $8 \%$ \\
\hline Estrutura Organizacional & 36 & $7 \%$ \\
\hline Simulação Virtual & 29 & $6 \%$ \\
\hline VSM & 28 & $5 \%$ \\
\hline Gestão Visual & 28 & $5 \%$ \\
\hline SBCE - Engenharia Simultânea baseada em conjuntos & 18 & $3 \%$ \\
\hline TOTAL & & $100 \%$ \\
\hline \multicolumn{1}{c|}{519} & &
\end{tabular}




\section{Metodologia Survey, Resultados e Discussões}

A metodologia utilizada caracteriza-se como um levantamento via questionário. A amostra não é significativa, mas é usada como um teste piloto, na qual realizou-se posteriormente pesquisa-ação numa das empresas.

Nessa etapa, sete grandes empresas do Brasil responderam o questionário, sendo uma fabricante de calçados femininos, uma de roupas femininas, masculina, infanto-juvenil, moda casa e calçados para venda em varejo; uma do ramo de cama, mesa e banho; uma marca de meias, cuecas, lingeries, meias-calças e pijamas; uma fabricante de bancos automotivos; uma de calçados femininos e masculinos e outra com negócios hospitalares e de consumo (algodão, bandagem plástica, compressas).

Perguntou-se diretamente "O processo de desenvolvimento de produtos é padronizado?". No setor têxtil, $100 \%$ das respostas foram "sim". Em outros setores tais como bens de capital, bens de consumo, eletroeletrônico, autoindústria, siderurgia e metalurgia houve respostas negativas. Maiores detalhes da survey estão em Dal Forno [3], Dal Forno, Forcellini e Bornia [4].

Os indicadores identificados nas empresas são relacionados a tempo, qualidade e custo (Figura 1).

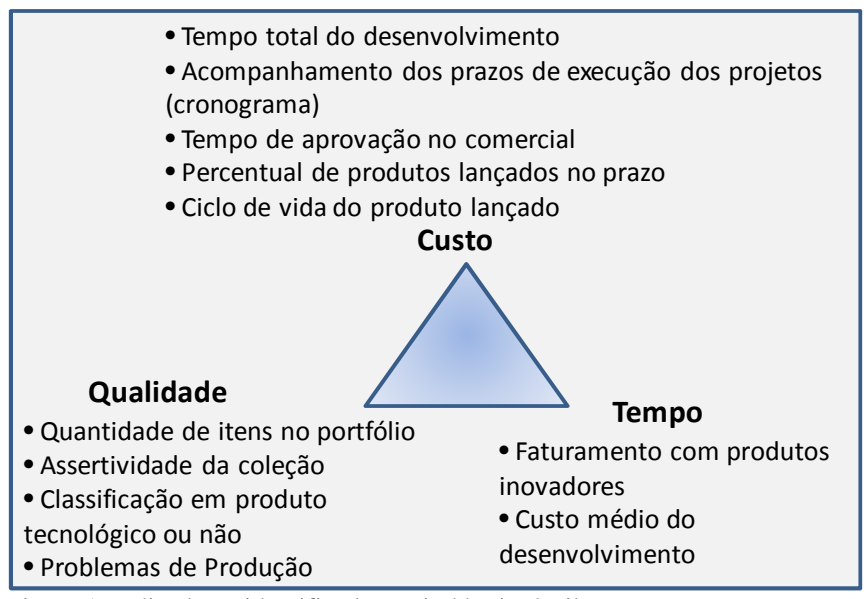

Figura 1. Indicadores identificados na indústria têxtil
A questão-chave, no entanto é - quais são os indicadores relacionados à padronização? Dal Forno, Miranda e Bornia [5] tentaram medir o grau de integração do processo de previsão de demanda através da identificação de como é realizado o fluxo de informações, como é a estrutura do processo, com qual frequência são gerados os relatórios e se há registro das lições aprendidas no Processo de Previsão de Demanda.

Assim, observa-se que não há especificamente um indicador em si para medir a padronização, mas sim sua periodicidade, ou seja, se é feito sempre da mesma maneira e periodicamente. Sejam os indicadores listados na Figura 1 ou outros, o importante é seguir uma estrutura, mostrar que há um planejamento do projeto, o que fortalece o que os autores enxutos destacam como reduzir a variabilidade das tarefas.

Seguindo a linha de Palmberg [11] pode-se afirmar que a padronização tem impacto nos demais indicadores, ou seja, um processo estruturado de desenvolvimento de produtos auxilia a reduzir custos e o tempo do desenvolvimento e melhorar a qualidade das informações.

No entanto, Morgan e Liker [10] entendem que o processo de desenvolvimento, do conceito até o lançamento do produto, possui muitas interações com o cliente. Logo, por mais que se tente padronizar, a cada projeto são os clientes, os tempos e as informações diferentes que influenciam nas decisões.

Posteriormente foram listados os desperdícios identificados no PDP. A Tabela 2 lista-os e classifica considerando a abordagem de Morgan e Liker [10].

Através desses desperdícios mencionados, observa-se que muitos deles são derivados da gestão, ou seja, da falta de fluxo e de uma definição de como precisa ser organizado o processo, em termos de tempo, qualidade e custo. Por exemplo, com a padronização do processo é possível minimizar os atrasos na execução dos cronogramas, alterações de requisitos após definições já estarem na fase de amostras, avaliações equivocadas e capacidades desbalanceadas. 
Tabela 2. Classificação dos desperdícios do PDP identificados na survey

\section{DESPERDÍCIOS}

\section{EXEMPLOS}

\begin{tabular}{|c|c|c|c|c|c|c|c|c|c|c|}
\hline $\begin{array}{l}\begin{array}{l}\text { Desenvolvimento feito de forma isolada por cada área, gerando } \\
\text { duplicidade: }\end{array} \\
\end{array}$ & $\mathrm{X}$ & & & $\mathrm{X}$ & & & $\mathrm{X}$ & $\mathrm{X}$ & $\mathrm{X}$ & \\
\hline Falta de comunicação entre os envolvidos & & $\mathrm{X}$ & & $\mathrm{X}$ & & $\mathrm{X}$ & & & & \\
\hline Falta de planejamento & & & & $\mathrm{X}$ & & & & & & $\mathrm{X}$ \\
\hline $\begin{array}{l}\text { Projetos avaliados de forma tecnicamente errada, acarretando prejuízos } \\
\text { financeiros }\end{array}$ & & & & & & $\mathrm{X}$ & & & $\mathrm{X}$ & \\
\hline Retrabalhos causados pelas modificações e ajustes nos projetos & & $\mathrm{X}$ & & & & $\mathrm{X}$ & & $\mathrm{X}$ & & \\
\hline Falta de análise no pré desenvolvimento & & & & $\mathrm{X}$ & & $\mathrm{X}$ & & & & $\mathrm{X}$ \\
\hline Falta de identificação dos requisitos do cliente & & & & $\mathrm{X}$ & & $\mathrm{X}$ & & & $\mathrm{X}$ & \\
\hline Atrasos na execução dos cronogramas & & $\mathrm{X}$ & & & & $\mathrm{X}$ & & & & \\
\hline Alterações nos requisitos do projeto & & & & & & $\mathrm{X}$ & & & $\mathrm{X}$ & \\
\hline Capacidade desbalanceada & & & & & & & & $\mathrm{X}$ & & $\mathrm{X}$ \\
\hline $\begin{array}{l}\text { O principal desperdício está no fluxo de informações entre Cliente, } \\
\text { Comercial e Engenharia. }\end{array}$ & & $\mathrm{X}$ & $X$ & & $\mathrm{X}$ & & & & & \\
\hline $\begin{array}{l}\text { Desperdício de capital intelectual, visto que em muitos casos a área de } \\
\text { Marketing aceita as sugestões depois de já ter sido testados }\end{array}$ & & & & & & $X$ & & & $X$ & $X$ \\
\hline
\end{tabular}

Desperdícios - (1) Superprodução, (2) Espera, (3) Transporte, (4) Processos Desnecessários, (5) Movimentação, (6) Defeitos, (7) Estoque, (8) Reinvenção, (9) Falta de disciplina, (10) Falta de Integração de TI.

As quatro práticas mais citadas na literatura - rede de aprendizado, voz do consumidor, envolvimento inicial do fornecedor e integração/engenharia simultânea confirmam-se através dos desperdícios mencionados pelas empresas têxteis. Então, algumas diretrizes para minimizar esses desperdícios são utilizar listas de verificação, mapear o processo e realizar as melhorias para obter o fluxo, aplicar a gestão visual, aplicar técnicas de voz do consumidor para captar as necessidades dos clientes, investir em treinamento da equipe tanto para nivelar conceitos quanto para desenvolver as habilidades de equipe de projeto multidisciplinar.

\section{Conclusão}

A padronização nem sempre aparece de forma explícita e direta nos trabalhos avaliados. Similarmente como afirmado na literatura, está associada com a rede de aprendizado. Isso pode ser devido à motivação para as melhorias realizadas através de kaizen e a preocupação em documentar as lições aprendidas. No outro extremo, a SBCE é a prática mais distante da padronização. Trabalhos futuros irão investigar a relação de outras práticas do desenvolvimento enxuto e seu impacto nos resultados obtidos além de detalhar o diagnóstico da padronização de processos.
Uma das explicações da prática padronização estar mais relacionada com a rede de aprendizado justifica-se na afirmação de Sobek [14] na qual a padronização é um ingrediente chave para a aprendizagem organizacional do sistema enxuto. Ela permite que a empresa estabeleça uma base para comparar as melhorias e assim enquadrá-la nas melhores práticas. A padronização é fundamental para o sistema da Toyota, pois é o principal mecanismo para capturar o conhecimento que é criado durante o desenvolvimento e compartilhá-lo com o resto da organização. As normas geradas também servem como um registro histórico das lições aprendidas, de modo que o conhecimento se expande ao longo do tempo e é disseminado para projetos futuros, Sobek [14].

Assim como na manufatura, esse artigo destacou a importância de começar a implementação do PDP enxuto pela prática de padronizar o processo. A revisão sistemática da literatura evidenciou que há potenciais de aplicação em diversos países e setores da economia. A survey destacou que há divergências de conceitos e que muitas empresas que desejam tornarem-se enxutas no desenvolvimento ainda nem possuem os processos definidos e entendidos de forma comum por todos que estão envolvidos. As aplicações podem ser expandidas para outros setores além do têxtil para verificar se esse é um problema comum em outras empresas. Para a academia, esse artigo 
auxiliou a diagnosticar que a padronização precisa ser investigada de forma indireta e não questionada diretamente.
[14] SOBEK, Durward K. A Toyota-Chrysler comparison. Dissertation for the degree of Doctor of Philosophy Industrial and Operations Engineering. University Michigan, 1997.

\section{Agradecimentos}

Os autores agradecem a CAPES - Coordenação de Aperfeiçoamento de Pessoal de Nível Superior pelo auxílio financeiro e à UNISC pelo FAP - Fundo de Apoio à Pesquisa.

\section{Referências}

[1] BAUCH, C. Lean Product Development: making waste transparent. Diploma thesis. Departament of Mechanical Engineering. Massachusetts Institute of Technology (MIT), jan 2004.

[2] CHAVES FILHO, José Geraldo Batista. Aplicação da padronização do método de trabalho segundo uma metodologia baseada na produção enxuta: um estudo de caso. Trabalho de Graduação. Departamento de Engenharia de Produção, Universidade Federal de São Carlos, São Carlos/SP, 2007.

[3] DAL FORNO, A. J. Método de Avaliação via benchmarking do Processo de Desenvolvimento Enxuto de Produtos. Tese. Doutorado em Engenharia de Produção. Florianópolis/SC: Universidade Federal de Santa Catarina, 2012.

[4] DAL FORNO, A.J. ; FORCELLINI, F. A. ; BORNIA, A. C. Lean Product Development: Benchmarking in Brazilian Companies. In: 22 International Congress of Mechanical Engineering (COBEM 2013), 2013, Ribeirão Preto/SP. Engineering for Technology Innovation, 2013.

[5] DAL FORNO, A. J.; MIRANDA, R. G. de.; BORNIA, A.C. Lean Demand: um método para diagnosticar a gestão da previsão de demanda de forma enxuta. In: XXXIV ENEGEP - Encontro Nacional de Engenharia de Produção. Curitiba: Abepro, 2014.

[6] DENNIS, P. Produção Lean Simplificada. Porto Alegre: Bookman, 2008.

[7] FERRO, José Roberto. Efetiva solução de problemas: o que está faltando? Lean Institute Brasil, 2006. Disponível em http://www.lean.org.br/leanmail/35/efetiva-solucao-de-problemas--o-queesta-faltando.aspx, acessado em 27/08/2014.

[8] GOH, Y. M.; MCMAHON, C. Improving reuse of in-service information capture and feedback. Journal of Manufacturing Technology Management, v.20, n.5, 2009, pp. 626-639.

[9] MAÇANEIRO, M. B.; CATCZU; T. S.; KORCHAQUE, E. de .L Assessoria executiva na padronização e organização de processos administrativos. Revista de Gestão e Secretariado - GeSec, São Paulo, v. 3, n. 1, p 109-133, jan./jun. 2012.

[10] MORGAN, J.; LIKER, J. K. Sistema Toyota de desenvolvimento de produto: integrando pessoas, processo e tecnologia. Trad. Raul Rubenich. Porto Alegre: Bookman, 2008.

[11] PALMBERG, K. Experiences of implementing process management: a multiple-case study. Business Process Management Journal, v.16, n.1, 2010, pp. 93-113.

[12] PERIN, P. C. (2005). Metodologia de padronização de uma célula de fabricação e de montagem, integrando ferramentas de produção enxuta. Dissertação (Mestrado) - Escola de Engenharia de São Carlos, Universidade de São Paulo, São Carlos, 2005.

[13] ROZENFELD, H. et al. Gestão de desenvolvimento de produtos: uma referência para a melhoria do processo. São Paulo: Saraiva, 2006.

\section{A PROCESS STANDARDIZATION AS THE FIRST STEP \\ FOR THE IMPLEMENTATION OF LEAN PRODUCT DEVELOPMENT - EVIDENCE OF TEXTILE INDUSTRY}

ABSTRACT: The lean approach emphasizes that standardization is the basis to begin with, ie, we need the stability of the processes and people. However, in practice it is noticed that many companies want to be "lean" is forgetting that initial preparation. Thus, this paper aims to analyze the standardization process in terms of bibliometrics, waste associated indicators and lean development practices associated with it. The methodology used was a systematic review of the literature, followed by survey with seven textile companies that develop products in Brazil. The results indicate that the product development process standardization is still a practice that needs to be explored further in business and academia, because it does not always appear in a direct manner and lack of understanding of its concept, benefits and problems may generate waste that impact the project scope and consequently usability.

Keywords: Textile. Standardization. Product development. Lean approach. 